\title{
Behavioral Treatments for Pediatric Insomnia
}

\author{
Eric S. Zhou ${ }^{1,2,3}$ • Judith Owens ${ }^{2,3}$
}

Published online: 9 August 2016

(C) Springer International Publishing AG 2016

\begin{abstract}
Pediatric insomnia, defined as chronic and clinically significant difficulty with initiating and/or maintaining sleep in children and adolescents, can profoundly impact functioning and well-being on both the child and their family. Behavioral interventions for insomnia in pediatric populations have been shown to be highly effective. This review expands upon this growing literature by summarizing the studies that have examined behavioral treatments for insomnia in children and adolescents published since 2013. Three themes emerge: (1) how pediatric insomnia is being treated in health settings; (2) efforts to adapt treatment to unique patient populations; and (3) the development of novel delivery methods for effective dissemination of treatment. Recommendations for important future clinical research questions are discussed.
\end{abstract}

Keywords Pediatric insomnia $\cdot$ Sleep disorders $\cdot$ Behavioral therapy $\cdot$ Cognitive-behavioral treatment

This article is part of the Topical Collection on Behavioral Therapy

Eric S. Zhou

eric zhou@dfci.harvard.edu

1 Dana-Farber Cancer Institute, 450 Brookline Ave, Boston, MA 02215, USA

2 Harvard Medical School, 25 Shattuck Street, Boston, MA 02115, USA

3 Boston Children's Hospital, 300 Longwood Ave, Boston, MA 02115, USA

\section{Introduction}

\section{Pediatric Insomnia}

Insomnia in children, similar to the definition in adults, is primarily characterized by difficulty initiating or maintaining sleep and/or poor sleep quality which results in significant impairments in daytime functioning. In contrast to adults, early morning awakening and nonrestorative sleep are far less common symptoms in children. Furthermore, it is often the caregiver rather than then the patient who offers the presenting complaints; in clinical practice, these are typically bedtime resistance, prolonged sleep onset, and/or night wakings which, especially in younger children, require parental intervention. Consequences related to pediatric insomnia not only include disruption of physical and psychological health $[1,2]$ but also meaningful functional impairments including poor school performance and behavioral dysfunction (e.g., oppositionality, defiance, and increased impulsivity) [3, 4]. In addition, caregivers of children with poor sleep must also bear a health burden as they are more likely to report depression, distress, fatigue, and marital conflict $[5,6]$.

It is estimated that up to $40 \%$ of typically developing children report insomnia symptoms [1, 7, 8], and the lifetime insomnia disorder prevalence for adolescents in the USA exceeds $10 \%$ [9]. Thus, due to the high prevalence and significant impact on both children and families, there has been an increasing level of interest among clinical researchers in identifying evidence-based treatment modalities for pediatric insomnia disorder, with a particular focus on behaviorally based interventions $[10,11]$. 


\section{Treatment for Pediatric Insomnia}

Multiple literature reviews, including a number of recently published comprehensive summaries of behavioral treatment of sleep disorders [12-15], have demonstrated that behavioral interventions are highly effective treatment modalities for insomnia, especially in young children $[16,17]$. These findings stand in contrast to the general lack of efficacy, as well as safety concerns, in studies examining pharmacologic treatment of insomnia in both children and adolescents $[18,19]$.

Key components of empirically based treatment for what was formally termed "behavioral insomnia of childhood" (sleep onset association and limit-setting subtypes) [20] include consistent bedtime routines, the development of appropriate sleep associations and self-soothing skills, bedtime fading (temporarily delaying bedtime to more closely approximate actual fall asleep time), decreasing parental attention for problematic bedtime behaviors, positive reinforcement for appropriate bedtime behaviors, and self-relaxation strategies [21].

It should be noted that while these strategies have considerable empirical support, concerns have been raised both by caregivers and by some clinicians and researchers regarding potential harm and cultural implications (e.g., extinction is likely to be inappropriate in cultures where co-sleeping is encouraged). A number of legitimate questions remain, but a recent comprehensive prospective controlled study examining the impact of standard behavioral interventions for insomnia in young children attempted to address many of these concerns (e.g., negative impact on attachment, psychological stress, and child function) found no evidence of long-term negative consequences on emotional, cognitive, and social functioning [22]. Furthermore, behavioral sleep interventions also appear to provide secondary benefits for both the children (e.g., improvements in self-esteem and emotional well-being [17] and the development of important self-regulation skills [23]) and their parents (e.g., mood, marital satisfaction [24, 25]).

In contrast, the literature examining effective treatment strategies for what was previously termed "primary" or "psychophysiologic" insomnia disorder in the pediatric population is sparse. While there is some controversy regarding how these evolving definitions of insomnia apply to the pediatric population, the more "adult-like" constellation of insomnia presentation symptoms characterized by heightened physiologic arousal, anxiety about the daytime consequences of insomnia maladaptive cognitions about sleep, and counterproductive behaviors to address insomnia (e.g., extending sleep when possible, daytime napping, lying in bed for extended periods awake) are rarely observed prior to age 10 years (with the exception of children with co-morbid anxiety [26]).

In adults, there exists a number of solid evidence-based approaches to the treatment of insomnia, including cognitive-behavioral therapy for insomnia (CBT-I), yoga, and mindfulness-based practices [27-30]. However, there are very limited data in pediatric populations, especially as some of the components of such treatments (e.g., cognitive re-appraisal, mindfulness meditation, etc.) are not fully appropriate for younger patients. In this developing field of research, efforts have increasingly focused on the application of strategies proven to be effective in adults in older children and adolescents [31-37].

The purpose of this review is to present a summary of recent literature published within the past 3 years relevant to the behavioral treatment of insomnia in children and adolescents. We identified pertinent publications in the period from 2013 to early 2016 by conducting a review of three major electronic bibliographic databases: PubMed, PsycINFO, and Google Scholar. Searches used the term "pediatric" as the main indicator, and combined this with either "insomnia," or "sleep" and searched using the following additional search terms: "behavioral," "behavioral treatment," "behavioral therapy," "cognitive," "cognitive treatment," "cognitive therapy," "therapy," and "treatment." In addition, we examined the references of the publications that were identified using these bibliographic databases, as well as searched for which newer publications had cited manuscripts published in the relevant time period by using Google Scholar's "cited by" feature. Three key themes appeared in the literature. First, researchers documented how pediatric insomnia is currently being treated in health settings. Second, there is increasing attention to adapting behavioral insomnia treatments within unique pediatric populations. Finally, there is a push to address treatment barriers by thinking of novel ways in which these evidence-based treatments can be effectively disseminated in community settings.

\section{Treatment of Pediatric Insomnia in Medical Settings}

As practice patterns in community clinical settings can vary, several studies have examined practice patterns regarding treatment of sleep problems in children. For example, Australian researchers conducted an online survey of 181 pediatricians and inquired about how they managed sleep disturbances [38]. Though the majority of pediatricians incorporated behavioral strategies in their management of sleep problems, the use of medication was more commonly reported. Results indicated that more than half of responding providers had prescribed melatonin to patients as young as 6 months of age, primarily for difficulty with sleep initiation and delayed sleep phase. Similarly, researchers in Canada concluded that $88.9 \%$ of physicians had recommended over-the-counter medications and $66.1 \%$ prescription medications for children with sleep problems within the past 6 months, especially in patients with developmental delays [39]. 
In the subspecialty setting of a pediatric behavioral sleep medicine clinic, Byars and Simon conducted a study of insomnia evaluation and treatment [40]. This study provided encouraging data demonstrating that CBT-I was effective in reducing insomnia symptoms on a center-specific insomnia severity measure by $40 \%$ in children and $29 \%$ in adolescents. Younger patients typically received parent-guided interventions that were behavioral in nature, whereas older patients responded well to CBT-I. Study authors also found that most behavioral interventions were relatively brief and timelimited; most did not last beyond 12 weeks and none required more than four clinical sessions. Approximately $90 \%$ of patients who formally terminated treatment with their provider reported improvements to their insomnia, compared with $80 \%$ in those who terminated early.

Inconsistencies in the delivery of clinical care can be problematic for patients. Thus, opportunities to provide further training for clinicians focused on the classification and appropriate treatment (both pharmacological and nonpharmacological) of sleep disorders in children would be helpful. For example, training packages for pediatric providers, including clinical decision flowcharts, are currently in development by the Australasian Sleep Association [38]. Furthermore, efforts to delineate the most effective delivery systems for children's sleep services, as well as efficacy trials of behavioral treatments in a variety of community settings (e.g., inner city and rural environments), and for a range of populations (e.g., racial/ethnic minorities, recent immigrants), will be key in addressing the full spectrum of pediatric patients.

\section{Adapting Behavioral Treatments for Insomnia in Special Pediatric Populations}

\section{Patients with Medical and Neurodevelopmental Conditions}

A number of recent studies have focused on identifying and addressing sleep problems in special populations, including children with Down syndrome [41, 42], autism spectrum disorders (ASD; [43-46]), developmental delays [47], cerebral palsy and epilepsy [48], migraine headaches [49-51], Tourette syndrome [52], Prader-Willi syndrome [53], Williams syndrome [54], and neurofibromatosis type 1 [55], among others [56-59]. The emotional and behavioral deficits that are at the core of many developmental disorders may impede efforts to successfully treat insomnia among these patient populations. However, it should be noted that recent efforts to deliver insomnia treatment in these populations have largely been successful, highlighted by a flexible approach with frequent follow-up. Though these trials included small sample sizes, the work is important because behavioral treatment for insomnia is commonly considered front-line therapy in typically developing children, and there is a need for further evidence supporting its feasibility and acceptance for parents and providers in populations with special needs $[60,61]$.

One study involved five children with a diagnosis of Angelman syndrome (a genetic syndrome characterized by severe speech impairment, intellectual disability, and motor coordination problems) [62]. The behavioral treatment approach instructed the child's parents to (1) create a sleepcompatible environment, (2) adjust and regulate the sleepwake schedule, and (3) manage parent-child interactions to reinforce appropriate sleep-related behaviors, particularly during the initiation phase. To address possible concerns about treatment adherence, a project coordinator maintained contact with the families on a weekly basis via telephone and email, and parents were encouraged to contact the study team during treatment as often as necessary. In addition, treatment duration was flexible, depending on the child's response to the intervention. Over the first month of treatment, disruptive behaviors decreased to almost zero and the children all improved in their ability to fall asleep independently. As a result, sleep duration increased an average of $30 \mathrm{~min}$ per night.

In another case study, two boys who had been diagnosed with ASD were successfully treated for insomnia symptoms using an individualized and comprehensive treatment approach [63]. One of the boys engaged in stereotypy (repetitive head shaking, body rocking, etc.) that delayed sleep onset, and repetitive item manipulation that was associated with extended night awakenings. The second boy struggled with selfsoothing that caused both sleep onset and maintenance difficulties. In both cases, basic behavioral strategies were implemented by clinicians, as well as specific case-related intervention adaptations (e.g., restricting access to stereotypy reinforcers), and using a video monitor to determine when to interrupt sleep interfering behaviors. Results demonstrated the efficacy of this individualized treatment model, with the study authors emphasizing the importance of a comprehensive evaluation of factors influencing each child's sleep problems.

Other researchers have targeted parents in order to improve the patient's sleep. Malow et al. [64] conducted a randomized trial of individual versus group-based sleep education for parents of 80 children between 2 and 10 years of age with ASD. The sleep education curriculum discussed specific components of successful sleep and behavioral strategies to improve sleep. It was delivered either via one 1-h individual phone call with two follow-up phone calls or two 2-h group sessions conducted over 2 weeks and followed up with two phone calls. Study findings revealed that delivery method did not significantly affect sleep and behavior outcomes. Across individual- and group-based approaches, the intervention was successful at improving actigraphy-measured sleep latency by almost $20 \mathrm{~min}$, and in self-reported questionnaire measures of sleep function. Another study of 28 children (5- 
13 years of age) with co-morbid ASD and attention-deficit/ hyperactivity disorder (ADHD) included a two-session (plus follow-up phone call) intervention which discussed behavioral insomnia treatment strategies and sleep hygiene [65]. Compared to usual care, the intervention group reported improvements in their sleep, as well as behavioral functioning, up to 6 months later. However, a parent-focused intervention which was longer in duration (five sessions) did not result in statistically significant improvements to sleep outcomes in 40 families with young children diagnosed with ASD [66]. Study authors suggested that their null results may have been due to inclusion criteria that were too broad and a limited sample size.

Another patient population that has been the focus of recent research is childhood cancer patients and their families. For example, children undergoing inpatient chemotherapy slept over $35 \mathrm{~min} / \mathrm{night}$ less and had poorer sleep efficiency than healthy, age-matched controls as measured by actigraphy [67], and parents reported significantly less total sleep, and more wake after sleep onset. [68]. Unfortunately, these problems often do not resolve once the patients leave the hospital. Matthews et al. [69] examined sleep in families of children during maintenance treatment for acute lymphoblastic leukemia, during which children routinely receive corticosteroid injections that have the potential to negatively impact sleep [70]. The study found that mothers of the children not only slept worse than controls but also perceived their insomnia symptoms to have a greater impact on daytime function. Like their caregivers, adolescent and young adult cancer survivors are likely to report disrupted sleep, with resulting impact on their physical and psychosocial health [71-73]. There have been recent efforts to target the sleep of these older survivors. Zhou et al. successfully delivered an adapted form of CBT-I to 12 childhood cancer survivors over three sessions, both in-person and over videoconference [74].

\section{Patients with Psychiatric Co-morbidities}

Recent research has also focused on whether CBT-I can augment cognitive-behavioral treatment for depression in adolescent patients (12-20 years of age) [75]. This pilot study found that 10 sessions of dual insomnia and depression treatment was effective at improving both objectively and subjectively measured insomnia symptoms, as well as some depression outcomes. These findings are not unexpected given previous studies reporting that pre-existing insomnia independently predicts the onset of depression in adolescents [76], and suggests that pairing an insomnia treatment in a mood disordered pediatric population is not only feasible but also may be advisable for some patients.

Next, given how common disrupted sleep is in children with ADHD [77], a number of recent studies have investigated the impact of behavioral interventions on insomnia in this group. Keshavarzi et al. [78] delivered a structured sleep program to children aged $8-13$ years with $\operatorname{ADHD}(N=40)$, which focused on monitoring sleep (sleep diaries), sleep scheduling, developing relaxing bedtime rituals, reducing electronics use, discussing positive events that occurred during the day or that the child was looking forward to, and developing a reward system for good sleep [79]. Parents reported significantly improved sleep, mood function, and social relationship function with family and peers following the intervention. In addition, the authors noted that improving the child's sleep also resulted in improvements in hyperactivity and/or conduct problems in their sample. Vetrayan et al. examined the impact of a 4-week behavioral sleep program on six children (aged 6-12 years) who were actively taking stimulant medications for their ADHD symptoms [80]. The intervention was effective in reducing sleep onset latency and bedtime resistance, and parents viewed the treatment as a positive experience.

These efforts to evaluate behavioral treatments for insomnia in special pediatric populations are a critical first step. However, many of these trials have required extensive provider involvement that would not be feasible in the average primary care or subspecialty clinical setting. Thus, implementation strategies that take into account the realities of clinical practice, including ways to manualize treatment so that other providers (e.g., social workers, nurses, etc.) can deliver therapy, or the leveraging technological advancements such as telemedicine may help to disseminate the findings from these efficacy trials.

\section{Novel Approaches to Insomnia Treatment Delivery}

Several barriers to the delivery of behavioral interventions for insomnia in pediatric populations, especially in primary care settings, have been identified. For example, an implementation of behavioral interventions for insomnia in children in the typical busy primary care clinical practice is challenging. In addition, the lack of availability of subspecialty (behavioral sleep medicine) care and an insufficient number of professionals with specialized training in sleep disorders all create problems with access to evidence-based care [81].

One area of interest has been to develop approaches which are embedded within the school setting as this has the potential to improve access and reduce the likelihood of study dropout $[34,82]$. Bei et al. offered a six-session program which delivered traditional components of CBT-I plus mindfulness-based practices to ten 13-15-year-old female adolescents in the school setting at the conclusion of the school day [83]. The program helped to improve both objective and subjective measures of sleep, and the intervention had an excellent completion rate. Another group of researchers sought to address possible concerns about low adolescent patient motivation to 
improve sleep and incorporated motivational interviewing techniques and parental involvement into their treatment approach [84]. Bonnar et al. studied 193 students in Grade 11 and also demonstrated that the school-based intervention was effective at improving sleep knowledge and outcomes. The authors noted that providing the participant's parents with access to study intervention materials (in lieu of direct face-toface intervention) did not significantly improve intervention efficacy. Finally, Paavonen et al. piloted a brief (four-session) behavioral sleep training program delivered to 16-18-year-old adolescents at school [85]. Actigraphic and subjective data revealed an increase in total sleep time (without a concomitant decrease in sleep efficiency) and improvements in overall subjective sleep quality. Furthermore, the authors also noted improvements in perceived stress and anxiety levels, even though the intervention did not target these outcomes specifically.

The adaptation of treatments for specific age groups has also been investigated. The Jugendlichen Schlaftraining (adolescent sleep training; JuSt) program, designed for adolescents between 11 and 17 years of age [86], consists of six weekly group sessions for up to eight patients, with five of the sessions delivered to the adolescents, with one session designed for parents. Key treatment components include psychoeducation about sleep, behavioral strategies to improve sleep, cognitive therapy addressing maladaptive sleep-related cognitions, guided imagery for relaxation, and hypnosis. A total of 19 participants completed the study, with results indicating significant improvements in sleep onset latency, wake after sleep onset, and sleep efficiency that were maintained across a 12-month follow-up period.

Another novel approach that has received increasing interest is the use of the Internet to deliver insomnia treatment in order to address several of the potential access barriers for patients [87]. Several efforts have been undertaken in the past several years to deliver Internet-based treatment in younger patients [88, 89]. More recently, Speth et al. delivered a 5session program that was adapted with age-specific information for toddlers (1-2 years), preschoolers (3-5 years), and school-aged children (6-10 years) [90]. Intervention content was delivered in text and through videos, and was directed at the child's parents. Their pilot findings indicated that an important factor influencing a parents' decision to use an Internet intervention for their child's insomnia was whether their health professional "bought in" to a behavioral and online treatment approach. In addition, de Bruin et al. recently conducted a randomized controlled trial of a 6-week in-person CBT-I group therapy program adapted for adolescents compared with 6-week structured Internet therapy and waitlist control in adolescents 12-19 years of age [91, 92]. Participants in both the in-person and Internet therapy protocols reported significant improvements in both objective (actigraphy) and subjective measures of sleep onset latency, wake after sleep onset, total sleep time, and sleep efficiency compared with the waitlist control. The authors reported high treatment adherence, but did note that all study participants (including those who received Internet therapy) met face-toface with a study clinician prior to the start of treatment in order to establish a therapeutic contact, and that this may have been an important contributor to efficacy of the treatments.

Finally, it should be noted that, while promising, the untapped potential of delivering web-based treatment programs must be tempered by the challenges of this format. The development of an Internet intervention is costly, and usability issues related to technological errors and a lack of in person support may be an issue [90]. In addition, focus group data from 14-19-year-old adolescents indicate that some patients may prefer in-person therapy, rather than the use of an Internet-based approach [93].

\section{Future Directions}

While there is solid evidence to support the efficacy of behavioral treatments for pediatric insomnia in real-world clinical settings [40], much work remains to be done in developing the best evidence-based care for as many patients with pediatric insomnia as possible. For example, given how often insomnia is encountered in the primary care setting [94, 95], pediatricians may benefit from further training "around the classification and subsequent management of behavioral sleep difficulties" [38]. Efforts could include online training and involve the development of clinical decision flowcharts that could assist pediatricians in the ongoing care of their patients. It is also important to acknowledge that "one size does not fit all" in regard to effective insomnia treatment [96]; this is even more salient in the pediatric population in whom there are both important developmental changes and inter-individual differences in sleep over time. Thus, it is critical that a clinician have a systematic and thorough approach to evaluating both the child with insomnia and the family prior to the initiation of treatment [97] in order to best match the treatment modality with the given clinical situation.

Pediatric sleep researchers share many of the same challenges as clinicians in developing evidence-based behavioral interventions for insomnia. Current gaps include the following:

\section{Behavioral intervention content}

- Greater efforts are needed to understand how specific components of "all-in-one [behavioral] sleep solutions," and different treatment program durations and delivery formats may differentially impact sleep in children [98], similar to recent efforts to "fine-tune" CBT-I in adults [99]. 
- While there is evidence to suggest that electronics use is prevalent in children and negatively impacts sleep [100-102], interventions have not yet fully incorporated this message into their content.

\section{Target populations}

- More research is needed in patient groups with medical co-morbidities (e.g., those with developmental delays or chronic illness). Despite the prevalence of insomnia symptoms in patients with special needs, there is an unfortunate lack of evidence validating the use of standard, compared with adapted, behavioral interventions in this group [103].

- Almost all clinical trials have focused on delivering intervention content to mothers, rather than fathers. A recent editorial has indicated that increasing paternal involvement is relevant to advancing this field of research [104].

- Minority children, especially those who reside in urban environments, are at greater risk for the development of insomnia. This increased prevalence may be the result of environmental factors such as sleeping in overcrowded, unsafe, and noisy conditions [105], child physical and mental health issues [106, 107], and a variety of family and parenting factors [108]. Further research to address this growing health disparity is necessary in order to improve our ability to intervene with this at-risk patient population.

\section{Intervention delivery}

- More research is needed to assess the impact of different delivery formats on behavioral interventions for pediatric insomnia. For some patients, the use of a briefer and less intensive approach may be sufficient, and would result in improved adherence; other patients may require a more comprehensive model which could potentially include parental involvement (even with an older adolescent). This notion of a stepped-care model for insomnia treatment has been discussed in the adult literature [109] and warrants further research in pediatric patients as well [110].

- It will also be important to explore the feasibility, acceptability, and effectiveness of telehealth approaches to pediatric insomnia treatment, and for which types of patients and families this approach is likely to be successful.

- As insomnia has been shown to be associated with social and academic adverse outcomes in children (and vice versa), children's sleep problems and their struggles at school must be viewed as related [7]. In addition, it is critical to further explore the school setting as an opportunity to intervene with sleep [82], particularly as there may also be additional benefits with respect to school performance [111].
Intervention outcomes

- Future research in pediatric insomnia should also include an expanded repertoire of both subjective (e.g., validated questionnaires for all age groups and adapted for special populations) and objective outcome measures of sleep, including clinically meaningful biomarkers such as cardiovascular parameters, inflammatory markers, and physiologic stress measures [112, 113].

\section{Alternatives/adjuncts to behavioral treatment}

- While there are clinical circumstances in which the pharmacological treatment of insomnia in pediatric patients in combination with behavioral interventions may be beneficial, data regarding the safety and efficacy of such combined treatment modalities is currently lacking [114-118].

- Although complementary and alternative approaches to the treatment of insomnia in children show promise, there as yet have been only a few studies published. Carotenuto et al. used an acupressure wristband (originally designed to improve motion sickness/nausea symptoms) in adolescents with insomnia and demonstrated PSG-measured improvements in sleep onset latency, wake after sleep onset, and sleep efficiency [119]. Cone et al. piloted the use of healing touch in pediatric burn patients and noted improvements to the total sleep time and sleep efficiency [120].

\section{Conclusion}

Between 2013 and 2016, an ever increasing body of evidence further improved our understanding of behavioral treatment of insomnia in the pediatric population. These findings highlight the diversity, and therein the challenges, of this work. Significant attention has been paid to demonstrating the efficacy of behavioral treatments in medical and psychiatric populations, underscoring the importance of tailoring therapeutic content to the unique needs of different patient groups. Beyond the generalized challenges associated with working with a specific patient population are the specific intricacies of individual patient presentation. Each child with insomnia symptoms presents in a distinctive fashion, with their own environmental context and differing levels of family engagement. Recent efforts to adapt treatment protocols to meet these distinctive needs, as well as to improve treatment accessibility, have been promising. 


\section{Compliance with Ethical Standards}

Conflict of Interest Eric S. Zhou and Judith Owens declare that they have no conflict of interest.

Human and Animal Rights and Informed Consent This article does not contain any studies with human or animal subjects performed by any of the authors.

\section{References}

1. Roberts RE, Roberts CR, Duong HT. Chronic insomnia and its negative consequences for health and functioning of adolescents: a 12-month prospective study. J Adolesc Health. 2008;42(3):294 302.

2. Vriend J, Davidson F, Rusak B, Corkum P. Emotional and cognitive impact of sleep restriction in children. Sleep Med Clin. 2015;10(2):107-15.

3. Beebe DW. Cognitive, behavioral, and functional consequences of inadequate sleep in children and adolescents. Pediatr Clin North Am. 2011;58(3):649-65.

4. Shochat T, Cohen-Zion M, Tzischinsky O. Functional consequences of inadequate sleep in adolescents: a systematic review. Sleep Med Rev. 2014;18(1):75-87.

5. Meltzer LJ, Mindell JA. Relationship between child sleep disturbances and maternal sleep, mood, and parenting stress: a pilot study. J Fam Psychol. 2007;21(1):67-73.

6. Kelly RJ, El-Sheikh M. Marital conflict and children's sleep: reciprocal relations and socioeconomic effects. J Fam Psychol. 2011;25(3):412-22.

7. Hochadel J, Frolich J, Wiater A, Lehmkuhl G, Fricke-Oerkermann L. Prevalence of sleep problems and relationship between sleep problems and school refusal behavior in school-aged children in children's and parents' ratings. Psychopathology. 2014;47(2): $119-26$.

8. Stein MA, Mendelsohn J, Obermeyer WH, Amromin J, Benca R. Sleep and behavior problems in school-aged children. Pediatrics. 2001;107(4):E60.

9. Johnson EO, Roth T, Schultz L, Breslau N. Epidemiology of DSMIV insomnia in adolescence: lifetime prevalence, chronicity, and an emergent gender difference. Pediatrics. 2006;117(2):e247-56.

10. Brown KM, Malow BA. Pediatric insomnia. Chest. 2016;149(5): $1332-9$

11. Miano S, Peraita-Adrados R. Pediatric insomnia: clinical, diagnosis, and treatment. Rev Neurol. 2014;58(1):35-42.

12. Gruber R, Carrey N, Weiss SK, et al. Position statement on pediatric sleep for psychiatrists. J Can Acad Child Adolesc Psychiatry. 2014;23(3):174.

13. Allen SL, Howlett MD, Coulombe JA, Corkum PV. ABCs of sleeping: a review of the evidence behind pediatric sleep practice recommendations. Sleep Med Rev. 2015;29:1-14.

14. Lewin DS. Application of cognitive behavioral therapy for insomnia in the pediatric population. Sleep Med Clin. 2014;9(2):169 80.

15. Kuhn BR. Practical strategies for managing behavioral sleep problems in young children. Sleep Med Clin. 2014;9(2):181-97.

16. Kuhn BR, Elliott AJ. Treatment efficacy in behavioral pediatric sleep medicine. J Psychosom Res. 2003;54(6):587-97.

17. Mindell JA, Kuhn B, Lewin DS, Meltzer LJ, Sadeh A. Behavioral treatment of bedtime problems and night wakings in infants and young children. Sleep. 2006;29(10):1263-76.
18. Sangal RB, Blumer JL, Lankford DA, Grinnell TA, Huang H. Eszopiclone for insomnia associated with attention-deficit/hyperactivity disorder. Pediatrics. 2014;134(4):e1095-103. doi:10.1542 /peds.2013-4221.

19. Blumer JL, Findling RL, Shih WJ, Soubrane C, Reed MD. Controlled clinical trial of zolpidem for the treatment of insomnia associated with attention-deficit/ hyperactivity disorder in children 6 to 17 years of age. Pediatrics. 2009;123(5):e770-6.

20. American Sleep Disorders Association. International classification of sleep disorders: diagnostic and coding manual. Revised ed. Rochester, MA: ASDA; 1997.

21. Owens JA, Mindell JA. Pediatric insomnia. Pediatr Clin North Am. 2011;58(3):555-69.

22. Price AM, Wake M, Ukoumunne OC, Hiscock H. Five-year follow-up of harms and benefits of behavioral infant sleep intervention: randomized trial. Pediatrics. 2012;130(4):643-51.

23. Sadeh A, Mindell JA, Owens J. Why care about sleep of infants and their parents? Sleep Med Rev. 2011;15(5):335-7.

24. Hiscock H, Bayer J, Gold L, et al. Improving infant sleep and maternal mental health: a cluster randomised trial. Arch Dis Child. 2007;92(11):952-8.

25. Wolfson A, Lacks P, Futterman A. Effects of parent training on infant sleeping patterns, parents' stress, and perceived parental competence. J Consult Clin Psychol. 1992;60(1):41-8.

26. Alfano CA, Patriquin MA, De Los Reyes A. Subjective-objective sleep comparisons and discrepancies among clinically-anxious and healthy children. J Abnorm Child Psychol. 2015;43(7):1343-53.

27. Morin CM, Hauri PJ, Espie CA, et al. Nonpharmacologic treatment of chronic insomnia. An American academy of sleep medicine review. Sleep. 1999;22(8):1134-56.

28. Siebern AT, Manber R. Insomnia and its effective nonpharmacologic treatment. Med Clin North Am. 2010;94(3):581-91.

29. Ong JC, Manber R, Segal Z, et al. A randomized controlled trial of mindfulness meditation for chronic insomnia. Sleep. 2014;37(9): 1553.

30. Mustian KM. Yoga as treatment for insomnia among cancer patients and survivors: a systematic review. Eur Med J Oncol. 2013;1:106.

31. Bootzin RR, Stevens SJ. Adolescents, substance abuse, and the treatment of insomnia and daytime sleepiness. Clin Psychol Rev. 2005;25(5):629-44.

32. Haynes PL, Bootzin RR, Smith L, et al. Sleep and aggression in substance-abusing adolescents: results from an integrative behavioral sleep-treatment pilot program. Sleep. 2006;29(4):512-20.

33. Sousa D, Cortez I, Araujo JF, Azevedo D, Macedo CV. The effect of a sleep hygiene education program on the sleep-wake cycle of Brazilian adolescent students. Sleep Biological Rhythm. 2007;5(4): 251-8.

34. Cain N, Gradisar M, Moseley L. A motivational school-based intervention for adolescent sleep problems. Sleep Med. 2011;12(3):246-51.

35. Tan E, Healey D, Gray AR, Galland BC. Sleep hygiene intervention for youth aged 10 to 18 years with problematic sleep: a before-after pilot study. BMC Pediatr. 2012;12(1):1.

36. Schlarb AA, Liddle CC, Hautzinger M. JuSt - a multimodal program for treatment of insomnia in adolescents: a pilot study. Nat Sci Sleep. 2011;3:13-20.

37. Perfect MM, Elkins GR. Cognitive-behavioral therapy and hypnotic relaxation to treat sleep problems in an adolescent with diabetes. J Clin Psychol. 2010;66(11):1205-15.

38. Heussler H, Chan P, Price AM, et al. Pharmacological and nonpharmacological management of sleep disturbance in children: an Australian paediatric research network survey. Sleep Med. 2013;14(2):189-94.

39. Bock DE, Roach-Fox E, Seabrook JA, Rieder MJ, Matsui D. Sleep-promoting medications in children: physician prescribing 
habits in southwestern Ontario, Canada. Sleep Med. 2016;17:526.

40. Byars K, Simon S. Practice patterns and insomnia treatment outcomes from an evidence-based pediatric behavioral sleep medicine clinic. Clin Pract Pediatr Psychol. 2014;2(3):337.

41. Stores G, Stores R. Sleep disorders and their clinical significance in children with down syndrome. Dev Med Child Neurol. 2013;55(2):126-30.

42. Ashworth A, Hill CM, Karmiloff-Smith A, Dimitriou D. Cross syndrome comparison of sleep problems in children with down syndrome and williams syndrome. Res Dev Disabil. 2013;34(5):1572-80.

43. Baker E, Richdale A, Short M, Gradisar M. An investigation of sleep patterns in adolescents with high-functioning autism spectrum disorder compared with typically developing adolescents. Dev Neurorehabil. 2013;16(3):155-65.

44. Richdale AL, Baglin CL. Self-report and caregiver-report of sleep and psychopathology in children with high-functioning autism spectrum disorder: a pilot study. Dev Neurorehabil. 2015;18(4): 272-9.

45. Herrmann S. Counting sheep: sleep disorders in children with autism spectrum disorders. J Pediatr Health Care. 2016;30(2):143-54.

46. Humphreys JS, Gringras P, Blair PS, et al. Sleep patterns in children with autistic spectrum disorders: a prospective cohort study. Arch Dis Child. 2014;99(2):114-8.

47. Tietze A-L, Blankenburg M, Hechler T, et al. Sleep disturbances in children with multiple disabilities. Sleep Med Rev. 2012;16(2): $117-27$.

48. Ming X, Pak J, Mulvey MA, et al. Sleep complaints in cerebral palsy and/or epilepsy: a pediatric sleep questionnaire study. J Pediatr Neurol. 2014;12(3):127-35.

49. Perez-Villena A, Soto-Insuga V, Castano-De la Mota C, et al. The importance of sleep problems in children with headache and other neurodevelopmental disorders in neuropaediatric services. Rev Neurol. 2016;62(2):61-7.

50. Guidetti V, Dosi C, Bruni O. The relationship between sleep and headache in children: implications for treatment. Cephalalgia. 2014;34(10):767-76.

51. Bellini B, Panunzi S, Bruni O, Guidetti V. Headache and sleep in children. Curr Pain Headache Rep. 2013;17(6):335.

52. Ghosh D, Rajan PV, Das D, et al. Sleep disorders in children with Tourette syndrome. Pediatr Neurol. 2014;51(1):31-5.

53. Gibbs S, Wiltshire E, Elder D. Nocturnal sleep measured by actigraphy in children with prader-willi syndrome. J Pediatr. 2013;162(4):765-9.

54. Axelsson EL, Hill CM, Sadeh A, Dimitriou D. Sleep problems and language development in toddlers with williams syndrome. Res Dev Disabil. 2013;34(11):3988-96.

55. Licis AK, Vallorani A, Gao F, et al. Prevalence of sleep disturbances in children with neurofibromatosis type 1. J Child Neurol. 2013;28(11):1400-5.

56. Angriman M, Caravale B, Novelli L, Ferri R, Bruni O. Sleep in children with neurodevelopmental disabilities. Neuropediatrics. 2015;46(3):199-210.

57. Barrett JR, Tracy DK, Giaroli G. To sleep or not to sleep: a systematic review of the literature of pharmacological treatments of insomnia in children and adolescents with attention-deficit/hyperactivity disorder. J Child Adolesc Psychopharmacol. 2013;23(10):640-7.

58. Corkum P, Davidson FD, Tan-MacNeill K, Weiss SK. Sleep in children with neurodevelopmental disorders: a focus on insomnia in children with ADHD and ASD. Sleep Med Clin. 2014;9(2): $149-68$

59. Li Z, Thompson LA, Gross HE, Shenkman EA, Reeve BB, Dewalt DA, et al. Longitudinal associations among asthma control, sleep problems, and health-related quality of life in children with asthma: a report from the Promis ${ }^{\circledR}$ pediatric asthma study. Sleep Med. 2016;20:41-50.

60. Meltzer LJ, Mindell JA. Systematic review and meta-analysis of behavioral interventions for pediatric insomnia. J Pediatr Psychol. 2014;39(8):932-48.

61. Grigg-Damberger M, Ralls F. Treatment strategies for complex behavioral insomnia in children with neurodevelopmental disorders. Curr Opin Pulm Med. 2013;19(6):616-25.

62. Allen KD, Kuhn BR, DeHaai KA, Wallace DP. Evaluation of a behavioral treatment package to reduce sleep problems in children with angelman syndrome. Res Dev Disabil. 2013;34(1):676-86.

63. Jin CS, Hanley GP, Beaulieu L. An individualized and comprehensive approach to treating sleep problems in young children. J Appl Behav Anal. 2013;46(1):161-80.

64. Malow BA, Adkins KW, Reynolds A, et al. Parent-based sleep education for children with autism spectrum disorders. J Autism Dev Disord. 2014;44(1):216-28.

65. Papadopoulos N, Sciberras E, Hiscock H, Mulraney M, McGillivray J, Rinehart N. The efficacy of a brief behavioral sleep intervention in school-aged children with ADHD and comorbid autism spectrum disorder. J Atten Disord. 2015. doi:10.1177 $/ 1087054714568565$

66. Johnson CR, Turner KS, Foldes E, et al. Behavioral parent training to address sleep disturbances in young children with autism spectrum disorder: a pilot trial. Sleep Med. 2013;14(10):995-1004.

67. Setoyama A, Ikeda M, Kamibeppu K. Objective assessment of sleep status and its correlates among hospitalized children with cancer: an exploratory study. Pediatr Int. 2016. doi:10.1111/ped.12927.

68. McLoone JK, Wakefield CE, Yoong SL, Cohn RJ. Parental sleep experiences on the pediatric oncology ward. Support Care Cancer. 2013;21(2):557-64.

69. Matthews EE, Neu M, Cook PF, King N. Sleep in mother and child dyads during treatment for pediatric acute lymphoblastic leukemia. Oncol Nurs Forum. 2014;41(6):599-610.

70. Drozdowicz LB, Bostwick JM. Psychiatric adverse effects of pediatric corticosteroid use. Mayo Clinic Proceedings. 2014;89(6): 817-34.

71. Daniel L, Kazak AE, Li Y, et al. Relationship between sleep problems and psychological outcomes in adolescent and young adult cancer survivors and controls. Support Care Cancer. 2016;24(2):539-46.

72. Zhou ES, Manley PE, Marcus KJ, Recklitis CJ. Medical and psychosocial correlates of insomnia symptoms in adult survivors of pediatric brain tumors. J Pediatr Psychol. 2015;41(6):623-30.

73. Zhou ES, Recklitis CJ. Insomnia in adult survivors of childhood cancer: a report from project reach. Support Care Cancer. 2014;22(11):3061-9.

74. Zhou ES, Vrooman LM, Manley PE, Crabtree VM, Recklitis CJ. Adapted delivery of cognitive-behavioral treatment for insomnia in adolescent and young adult cancer survivors: a pilot study. Behav Sleep Med. 2016;14:1-14.

75. Clarke G, McGlinchey EL, Hein K, et al. Cognitive-behavioral treatment of insomnia and depression in adolescents: a pilot randomized trial. Behav Res Ther. 2015;69:111-8.

76. Alvaro PK, Roberts RM, Harris JK. The independent relationships between insomnia, depression, subtypes of anxiety, and chronotype during adolescence. Sleep Med. 2014;15(8):934-41.

77. Owens J, Gruber R, Brown T, et al. Future research directions in sleep and adhd report of a consensus working group. J Atten Disord. 2013;17(7):550-64.

78. Keshavarzi Z, Bajoghli H, Mohamadi MR, et al. In a randomized case-control trial with 10 -years olds suffering from attention deficit/hyperactivity disorder (ADHD) sleep and psychological functioning improved during a 12-week sleep-training program. World J Biol Psychiatry . 2014;15(8):609-19.

79. Fricke L, Mitschke A, Wiater A, Lehmkuhl G. A new treatment program for children with sleep disorders - concept, practicability, 
and first empirical results. Prax Kinderpsychol Kinderpsychiatr. 2005;55(2):141-54.

80. Vetrayan J, Othman S, Paulraj SJ V. Case series: evaluation of behavioral sleep intervention for medicated children with ADHD. J Atten Disord. 2013. doi:10.1177/1087054713479665.

81. Boerner KE, Coulombe JA, Corkum P. Barriers and facilitators of evidence-based practice in pediatric behavioral sleep care: qualitative analysis of the perspectives of health professionals. Behav Sleep Med. 2015;13(1):36-51.

82. Moseley L, Gradisar M. Evaluation of a school-based intervention for adolescent sleep problems. Sleep. 2009;32(3):334-41.

83. Bei B, Byrne ML, Ivens C, et al. Pilot study of a mindfulnessbased, multi-component, in-school group sleep intervention in adolescent girls. Early Interv Psychiatry. 2013;7(2):213-20.

84. Bonnar D, Gradisar M, Moseley L, et al. Evaluation of novel school-based interventions for adolescent sleep problems: does parental involvement and bright light improve outcomes? Sleep Health. 2015;1(1):66-74.

85. Paavonen EJ, Huurre T, Tilli M, Kiviruusu O, Partonen T. Brief behavioral sleep intervention for adolescents: an effectiveness study. Behav Sleep Med. 2015;14(4):1-16.

86. Roeser K, Schwerdtle B, Kübler A, Schlarb A. Further evidence for the just program as treatment for insomnia in adolescents: results from a 1-year follow-up study. J Clin Sleep Med. 2015;12(2):257-62.

87. Seyffert M, Lagisetty P, Landgraf J, et al. Internet-delivered cognitive behavioral therapy to treat insomnia: a systematic review and meta-analysis. PLoS One. 2016;11(2):e0149139.

88. Mindell JA, Du Mond CE, Sadeh A, et al. Efficacy of an internetbased intervention for infant and toddler sleep disturbances. Sleep. 2011;34(4):451-8

89. Mindell JA, Du Mond CE, Sadeh A, et al. Long-term efficacy of an internet-based intervention for infant and toddler sleep disturbances: one year follow-up. J Clin Sleep Med. 2011;7(5):507-11.

90. Speth TA, Coulombe JA, Markovich AN, et al. Barriers, facilitators, and usability of an internet intervention for children aged 1 to 10 years with insomnia. Transl Issues Psychol Sci. 2015;1(1):16.

91. de Bruin EJ, Oort FJ, Bogels SM, Meijer AM. Efficacy of internet and group-administered cognitive behavioral therapy for insomnia in adolescents: a pilot study. Behav Sleep Med. 2014;12(3):235-54.

92. de Bruin EJ, Bogels SM, Oort FJ, Meijer AM. Efficacy of cognitive behavioral therapy for insomnia in adolescents: a randomized controlled trial with internet therapy, group therapy and a waiting list condition. Sleep. 2015;38(12):1913-26.

93. Conroy DA, Czopp AM, Dore-Stites D, Dopp RR, Armitage R, Hoban TF, et al. A pilot study on adolescents with depression and insomnia: qualitative findings from focus groups. Behav Sleep Med. 2015;8:1-17.

94. Meltzer LJ, Plaufcan MR, Thomas JH, Mindell JA. Sleep problems and sleep disorders in pediatric primary care: treatment recommendations, persistence, and health care utilization. J Clin Sleep Med. 2014;10(4):421-6.

95. Honaker SM, Meltzer LJ. Sleep in pediatric primary care: a review of the literature. Sleep Med Rev. 2016;25:31-9.

96. Cvengros JA. One size does not fit all: matching patients with insomnia treatment modality. Sleep Med. 2014;15(2):161-2.

97. Bruni O, Angriman M. Pediatric insomnia: new insights in clinical assessment and treatment options. Arch Ital Biol. 2015;153(2-3): $154-66$.

98. Spruyt K, Curfs LM. Non-pharmacological management of problematic sleeping in children with developmental disabilities. Dev Med Child Neurol. 2015;57(2):120-36.

99. Riemann D, Spiegelhalder K. Insomnia research - time for "finetuning”. Sleep Med Rev. 2015;23:89-90.
100. Engelhardt CR, Mazurek MO, Sohl K. Media use and sleep among boys with autism spectrum disorder, ADHD, or typical development. Pediatrics. 2013;132(6):1081-9.

101. Séguin D, Klimek V. Just five more minutes please: electronic media use, sleep and behaviour in young children. Early Child Dev Care. 2015;186(6):1-20.

102. Hale L, Guan S. Screen time and sleep among school-aged children and adolescents: a systematic literature review. Sleep Med Rev. 2015;21:50-8.

103. Richdale AL, Baker EK. Sleep in individuals with an intellectual or developmental disability: recent research reports. Curr Dev Disord Rep. 2014;1(2):74-85.

104. Sadeh A, Mindell, JA. Infant sleep interventions - methodological and conceptual issues. Sleep Med Rev. 2015. doi:10.1016/j. smrv.2015.11.002.

105. Spilsbury JC, Storfer-Isser A, Kirchner HL, et al. Neighborhood disadvantage as a risk factor for pediatric obstructive sleep apnea. J Pediatr. 2006;149(3):342-7.

106. Fagnano M, Bayer AL, Isensee CA, Hernandez T, Halterman JS Nocturnal asthma symptoms and poor sleep quality among urban school children with asthma. Acad Pediatr. 2011;11(6):493-9.

107. Benoit D, Zeanah CH, Boucher C, Minde KK. Sleep disorders in early childhood: association with insecure maternal attachment. J Am Acad Child Adolesc Psychiatry. 1992;31(1):86-93.

108. McDonald L, Wardle J, Llewellyn CH, van Jaarsveld CH, Fisher A. Predictors of shorter sleep in early childhood. Sleep Med. 2014;15(5):536- 40.

109. Espie CA. "Stepped care": a health technology solution for delivering cognitive behavioral therapy as a first line insomnia treatment. Sleep. 2009;32(12):1549-58.

110. Gradisar M, Richardson C. CBT-I cannot rest until the sleepy teen can. Sleep. 2015;38:12.

111. Fatnani RH, Minajagi SKB, Rao C, Kumar KS. Study of sleep problems and their association with scholastic performance in school going children. Indian J Child Health. 2015;2(4):01-4.

112. Markovich AN, Gendron MA, Corkum PV. Validating the children's sleep habits questionnaire against polysomnography and actigraphy in school-aged children. Front Psychiatry. 2014;5:188.

113. Mouthon AL, Huber R. Methods in pediatric sleep research and sleep medicine. Neuropediatrics. 2015;46(3):159-70.

114. Cronin SD, Gottschlich MM, Gose LM, Kagan RJ. Zolpidem and sleep in pediatric burn patients with attention deficit/hyperactivity disorder. Pediatr Nurs. 2015;41(3):132-4. 140

115. Stockmann C, Gottschlich MM, Healy D, et al. Relationship between zolpidem concentrations and sleep parameters in pediatric burn patients. J Burn Care Res. 2015;36(1):137-44.

116. Cortese S, Brown TE, Corkum P, et al. Assessment and management of sleep problems in youths with attention-deficithyperactivity disorder. J Am Acad Child Adolesc Psychiatry. 2013;52(8):784-96.

117. Robinson AA, Malow BA. Gabapentin shows promise in treating refractory insomnia in children. J Child Neurol. 2013;28(12): 1618-21.

118. Bahremand Z, Hashemian P, Moharreri F, Soltani E. Melatonin effects on sleep disorders in children with attention deficit hyperactivity disorder. Rev Clin Med. 2014;1(4):233-7.

119. Carotenuto M, Gallai B, Parisi L, Roccella M, Esposito M. Acupressure therapy for insomnia in adolescents: a polysomnographic study. Neuropsychiatr Dis Treat. 2013;9:157-62.

120. Cone L, Gottschlich M, Khoury J, Simakajornboon N, Kagan R. The effect of healing touch on sleep patterns of pediatric burn patients: a prospective pilot study. J Sleep Disord Treat Care. 2014;3(2):1-6. 